\begin{tabular}{|c|l|}
\hline Title & Stark spectroscopy at Balmer-a line of atomic hydrogen for measuring sheath electric field in a hy drogen plasma \\
\hline Author(s) & Nishiyama, Shusuke; Nakano, Haruhisa; Goto, Motoshi; Sasaki, Koichi \\
\hline Citation & $\begin{array}{l}\text { Journal of Physics D: A pplied Physics, 50(23), 234003 } \\
\text { https://doi.org/10.1088/1361-6463/aa6e86 }\end{array}$ \\
\hline Issue Date & 2017-07-01 \\
\hline Doc URL & http://hdl.handle.net/2115/77155 \\
\hline Type & article (author version) \\
\hline File Information & JPhysD-112396.R1.pdf \\
\hline
\end{tabular}

Instructions for use 


\title{
Stark spectroscopy at Balmer- $\alpha$ line of atomic hydrogen for measuring sheath electric field in a hydrogen plasma
}

\author{
Shusuke Nishiyama ${ }^{1}$, Haruhisa Nakano ${ }^{2}$, Motoshi Goto ${ }^{2}$, and \\ Koichi Sasaki ${ }^{1}$ \\ ${ }^{1}$ Division of Quantum Science and Engineering, Hokkaido University, Sapporo \\ 060-8628, Japan \\ ${ }^{2}$ National Institute for Fusion Science, Toki, Gifu 509-5292, Japan \\ E-mail: shu@eng.hokudai.ac.jp
}

\begin{abstract}
This paper reports a diode laser based system which is applicable to the measurement of electric field in the sheath region of a hydrogen plasma. The electric field is deduced from the Stark spectrum of the Balmer- $\alpha$ line of atomic hydrogen. Saturation spectroscopy with a Doppler-free spectral resolution is adopted to detect the Stark effects of the low energy states. We have demonstrated a detection limit of $10 \mathrm{~V} / \mathrm{cm}$, which is a sufficient sensitivity for investigating the structures of the sheath electric fields in low-temperature plasmas. We have discussed the detection limit, the measurement ambiguity, the spatial resolution, and the limitation of the developed method.
\end{abstract}

PACS numbers: $52.70 . \mathrm{Kz}, 32.60 .+\mathrm{i}, 32.30 . \mathrm{Jc}$

Keywords: electric field, Stark effect, saturation spectroscopy, sheath

Submitted to: J. Phys. D: Appl. Phys. 
Stark spectroscopy at Balmer- $\alpha$ line of atomic hydrogen for measuring sheath electric field in a hydrogen $p$

\section{Introduction}

The measurement of sheath electric field is a difficult task in low-temperature plasma diagnostics. An attempt was to measure the spatial distribution of the potential using an emissive probe [1-5]. However, emissive probes have problems in the limited spatial resolution and the considerable disturbance to the plasma. The potential distribution can be estimated by measuring the velocity distribution function of ions by laserinduced fluorescence under the assumption of a collisionless sheath [6-9]. However, since the potential is given by the integral of the electric field, it is not easy to deduce the electric field from the potential distribution. Since the lack of diagnostics causes the insufficient understanding on the structure of the sheath electric field, a low-cost, easy-to-use method is highly demanded in both the fields of plasma processing and fundamental physics of low-temperature plasmas.

In principle, the direct measurement of the sheath electric field is possible by Stark spectroscopy. The principle of Stark spectroscopy is the evaluation of the electric field from the energy level structure of an atom or a molecule which is perturbed by the electric field (Stark effects). In many cases, a laser-aided spectroscopic method is used for detecting the energy level structure. The difficulty of laser Stark spectroscopy is caused mainly by the trade-off between the sensitivity to a weak electric field and the difficulty in the detection of energy states. Highly excited states (or Rydberg states) have sensitive Stark effects to a weak electric field. However, Rydberg states are difficult to be detected since the transition probability decreases steeply with the principal quantum number.

In the history of laser Stark spectroscopy, the first successful measurement was carried out by optogalvano spectroscopy [10-13]. A problem of this method was a limitation in the discharge geometry since the excitation to a Rydberg state was detected via the electric response of the plasma. Laser-induced collisional fluorescence spectroscopy was successfully adopted to the sheath electric field measurement [14-18], but this method had a limitation in the gas pressure since the fluorescence after the collisional transition to a lower-lying state was detected to know the excitation to a Rydberg state. Laser optogalvano spectroscopy and laser-induced collisional fluorescence spectroscopy were used for detecting the Rydberg states with principal quantum numbers of $6-10$, resulting in the sensitivity of $\sim 200 \mathrm{~V} / \mathrm{cm}$ for the electric field measurement. Much more sensitive measurements with detection limits of 3-5 $\mathrm{V} / \mathrm{cm}$, which were realized by detecting Rydberg states with principal quantum numbers of 40-55, were demonstrated by laser-induced fluorescence-dip spectroscopy [19-26]. This method was ideal since it has no limitations in the discharge geometry and the gas pressure. However, laser-induced fluorescence-dip spectroscopy requires two pulsed tunable lasers and sophisticated experimental skills.

In this work, we changed our approach to the detection of the Stark effects of lowerlying states. The detection of a low-energy state is easy since it has a large transition probability. However, since the magnitude of the energy shift of the low-energy state 
by Stark effects is much smaller than that of a Rydberg state, the energy shift is buried under the resolution of usual spectroscopic methods. The resolution of the laser-aided spectroscopic detection of energy states is determined by the linewidth of a pulsed tunable laser and the Doppler broadening width of the transition line. For example, the linewidth of a pulsed tunable laser is $\sim 0.2 \mathrm{~cm}^{-1}(6 \mathrm{GHz})$ and the Doppler broadening width of the Balmer- $\alpha$ line of atomic hydrogen is approximately $10 \mathrm{GHz}(15 \mathrm{pm})$ at a temperature of $1000 \mathrm{~K}$. Our idea is to use a spectroscopic method having Doppler-free spectral resolution $[27,28]$. We employed saturation spectroscopy at the Balmer- $\alpha$ line of atomic hydrogen $[29,30]$. Since it is possible to construct the system of saturation spectroscopy by using a tunable single-mode diode laser, we can realize an easy-to-use system having an ultrafine resolution with a low experimental cost.

\section{Principles and theoretical Stark spectra}

\subsection{Principles of saturation spectroscopy}

The principle of saturation spectroscopy is described in a textbook in detail [31]. Briefly, it is possible to detect the transition line with a Doppler-free resolution by injecting a pump laser beam from the counter direction to the probe laser beam. The pump and probe beams are yielded from the same laser source. Since atoms in a plasma are moving randomly by the thermal motion, an atom which has a velocity in the parallel direction to the laser beams feels that the pump and probe beams have different wavelengths. This is the Doppler effect or the Doppler shift. Hence, if the atom feels that the probe beam is resonant, it is not excited by the pump beam. On the other hand, if the atom has no velocity in the parallel direction, it feels that the pump and probe beams have the same wavelength. Therefore, if the wavelength of the laser is resonant with the transition line and the pump beam is intense enough to have the saturation, a dip, which is called the Lamb dip, is formed at the line center of the Doppler-broadened absorption spectrum of the probe beam. Since the width of the dip is given by the homogeneous broadening, the dip spectrum or the saturation spectrum has a Doppler-free resolution.

The intensity of the probe beam that is transmitted through the plasma is given by

$$
I_{\mathrm{t}}(\nu)=I_{\mathrm{i}} \exp \left(-\alpha_{\mathrm{s}}(\nu) l\right) \simeq I_{\mathrm{i}}\left\{1-\alpha_{\mathrm{s}}(\nu) l\right\}
$$

where $I_{\mathrm{i}}$ and $I_{\mathrm{t}}$ are the intensities of the incident and transmitted probe beams, respectively, $\alpha_{\mathrm{s}}(\nu)$ is the absorption coefficient with the Lamb dips, $l$ is the absorption length, and $\nu$ is the laser frequency. The approximated expression is valid in the present experimental condition because $\alpha(\nu) l \ll 1$. When the pump beam is intense enough to have the saturation, the absorption coefficient is given by

$$
\alpha_{\mathrm{s}}(\nu)=\alpha_{0}(\nu)\left[1-\frac{S_{0}}{2} \frac{\left(\gamma_{\mathrm{s}} / 2\right)^{2}}{\left(\nu-\nu_{0}\right)^{2}+\left(\Gamma_{\mathrm{s}} / 2\right)^{2}}\right],
$$

where $\alpha_{0}(\nu)$ is the Doppler-broadened absorption coefficient when the saturation is negligible, $\nu_{0}$ is the line center frequency of a transition line, $S_{0}$ represents the saturation 
parameter which is proportional to the intensity of the pump beam $I_{\mathrm{p}}, \gamma_{\mathrm{s}}=\gamma \sqrt{1+S_{0}}$ with $\gamma$ being the homogeneous broadening width, and $\Gamma_{\mathrm{s}}=\left(\gamma+\gamma_{\mathrm{s}}\right) / 2$. This expression is valid when $S_{0} \ll 1$. The signal intensity of the saturation spectrum is the difference between $I_{\mathrm{t}}$ in the presence and absence of the pump beam, and it is given by

$$
\Delta I_{\mathrm{t}}(\nu)=\frac{1}{2} I_{\mathrm{i}} S_{0} \alpha_{0}(\nu) l \frac{\left(\gamma_{\mathrm{s}} / 2\right)^{2}}{\left(\nu-\nu_{0}\right)^{2}+\left(\Gamma_{\mathrm{s}} / 2\right)^{2}} .
$$

\subsection{Theoretical Stark spectra}

Figure 1 shows the energy level diagram of atomic hydrogen relevant to the Balmer- $\alpha$ line. The Balmer- $\alpha$ line is composed of seven fine structure components according to the selection rule. The principle of Stark spectroscopy is to compare the spectrum observed experimentally with that obtained by the theoretical calculation of the Stark effects. The theoretical calculation is based on solving the time-independent Schrödinger equation with the perturbation of the electric field [32]. In the case of atomic hydrogen, it is possible to obtain the accurate solution of the Schrödinger equation. The theoretical Stark spectra are shown in Fig. 2. Figure 2(a) was obtained in the $\pi$ polarization configuration where the polarizations of the laser beams are parallel to the electric field, while the theoretical Stark spectrum shown in Fig. 2(b) was obtained in the $\sigma$ polarization configuration where the polarizations of the laser beams are perpendicular to the electric field. The widths $(420 \mathrm{MHz})$ of the peaks are assumed by referring to the experimental result. The seven fine structure components are indicated by labels A-G in the spectrum in the absence of the electric field, although peaks A and E are too weak to be identified in this plot. The labels correspond to those indicated in the energy level diagram shown in Fig. 1. The origin of the horizontal axis of Fig. 2 is the frequency of the $2 \mathrm{p}^{2} \mathrm{P}_{3 / 2}^{\mathrm{o}}-3 \mathrm{~d}^{2} \mathrm{D}_{5 / 2}$ (peak $\mathrm{C}$ ) transition.

As shown in Fig. 2(a), in the $\pi$ polarization configuration, the peak frequencies of the $2 \mathrm{p}^{2} \mathrm{P}_{3 / 2}^{\mathrm{o}}-3 \mathrm{~d}^{2} \mathrm{D}_{5 / 2}$ (peak $\mathrm{C}$ ) and $2 \mathrm{p}^{2} \mathrm{P}_{1 / 2}^{\mathrm{o}}-3 \mathrm{~d}^{2} \mathrm{D}_{3 / 2}$ (peak $\mathrm{G}$ ) transitions shifted toward the high-frequency side with the increase in the electric field from 0 to $150 \mathrm{~V} / \mathrm{cm}$. In contrast, the frequencies of the $2 \mathrm{p}^{2} \mathrm{P}_{3 / 2}^{\mathrm{o}}-3 \mathrm{~d}^{2} \mathrm{D}_{3 / 2}$ (peak $\mathrm{B}$ ) and $2 \mathrm{~s}^{2} \mathrm{~S}_{1 / 2}-3 \mathrm{p}^{2} \mathrm{P}_{1 / 2}^{\mathrm{o}}$ (peak D) transitions shifted toward the low-frequency side. The linewidths of peaks $\mathrm{B}$ $\left(2 \mathrm{p}^{2} \mathrm{P}_{3 / 2}^{\mathrm{o}}-3 \mathrm{~d}^{2} \mathrm{D}_{3 / 2}\right)$ and $\mathrm{F}\left(2 \mathrm{~s}^{2} \mathrm{~S}_{1 / 2}-3 \mathrm{p}^{2} \mathrm{P}_{3 / 2}^{\mathrm{o}}\right)$ were broadened by Stark splitting, when the electric field was $200 \mathrm{~V} / \mathrm{cm}$. The splittings of peaks B and $\mathrm{C}$ were clearly observed at an electric field of $400 \mathrm{~V} / \mathrm{cm}$. At electric fields higher than $400 \mathrm{~V} / \mathrm{cm}$, peaks B and C with the splittings shifted roughly linearly with the electric field. The magnitudes of the shifts of peaks $\mathrm{F}$ and $\mathrm{G}$ were smaller than those of peaks B and $\mathrm{C}$. On the other hand, in the $\sigma$ polarization configuration, the positions of peaks $\mathrm{B}$ and $\mathrm{C}$ were not sensitive to the electric field. The broadening of peak $\mathrm{C}$ was more significant in the $\sigma$ polarization configuration, and peak $\mathrm{C}$ merged into peak $\mathrm{B}$ at an electric field of $200 \mathrm{~V} / \mathrm{cm}$. The splittings and the shits of peaks D and E were more sensitive to the electric field, but their amplitudes were quite small. The splittings and broadenings of peaks $\mathrm{F}$ and $\mathrm{G}$ were also observed, but the shifts of the peaks were not clear. 


\section{Stark spectroscopy at Balmer- $\alpha$ line of atomic hydrogen for measuring sheath electric field in a hydrogen $p$}

\section{Experiment}

The experimental apparatus is schematically shown in Fig. 3. An inductively-coupled plasma source with an internal rf antenna was used in this experiment. The antenna was connected to an rf power supply at $13.56 \mathrm{MHz}$ via a matching circuit. The rf power was pulse modulated at a frequency of $20 \mathrm{kHz}$. The instantaneous power and the duty factor were $1 \mathrm{~kW}$ and $50 \%$, respectively. The vacuum chamber was a stainless-steel cylinder which was grounded electrically. The inner diameter and the height of the vacuum chamber were $26 \mathrm{~cm}$. Pure hydrogen was fed into the chamber via a mass-flow controller at a flow rate of $100 \mathrm{sccm}$ to generate the discharge. The gas pressure was 70 mTorr. A rectangular planer electrode with a size of $5 \times 15 \mathrm{~cm}^{2}$ was installed in the chamber. The distance between the electrode and the $\mathrm{rf}$ antenna was $5 \mathrm{~cm}$. The electrode was supported by a micrometer, and was connected to a dc power supply. The electron density and the space potential of the plasma, which were measured using a Langmuir probe in the $\mathrm{cw}$ plasma without placing the electrode, were approximately $1 \times 10^{10} \mathrm{~cm}^{-3}$ and $8 \mathrm{~V}$, respectively. The electron density and the space potential in the pulsed plasma with the electrode could be different from these values.

A linearly-polarized, single-mode diode laser (New Focus TLB-6900) was used for constructing the system of saturation spectroscopy. The wavelength of the laser was scanned over the whole range of the Doppler broadened Balmer- $\alpha$ line of atomic hydrogen. The wavelength scan spent $10 \mathrm{~s}$. The relative frequency of the laser beam was monitored using a Fabry-Pérot spectrum analyzer with a free spectral range of $1 \mathrm{GHz}$. A small part of the laser beam was picked up using a beam sampler and was used as the probe beam. The probe beam was injected into a polarization-maintained single-mode optical fiber with a core diameter of $3.5 \mu \mathrm{m}$. The other part of the laser beam was amplified using a diode laser amplifier (Toptica BoosTA), and was used as the pump beam. The pump beam was injected into another polarization-maintained single-mode optical fiber. The output beams from the optical fibers were collimated using lenses, and were injected into the plasma from the counter directions. The probe and pump beams were parallel to the electrode surface. The diameters (FWHM) of the collimated probe and pump beams were approximately $0.3 \mathrm{~mm}$. The two beams were crossed with a small angle of approximately $10 \mathrm{mrad}$ above the center of the electrode. This optical alignment resulted in $3 \mathrm{~cm}$ for the overlapped length of the pump and probe beams. The distance between the electrode surface and the laser beams was adjusted by moving the electrode. The polarizations of the laser beams were parallel or perpendicular to the sheath electric field. The polarizations were changed by rotating the optical fibers. The powers of the probe and pump beams were 0.34 and $18.6 \mathrm{~mW}$, respectively. The probe beam passed through the plasma was introduced into a photodiode detector via an interference filter at the Balmer- $\alpha$ line. The electrical signal from the photodiode was connected to a lock-in amplifier to observe the synchronous component with the modulation frequency of the rf power. 
Stark spectroscopy at Balmer- $\alpha$ line of atomic hydrogen for measuring sheath electric field in a hydrogen $p$

\section{Results and discussion}

\subsection{Observation of fine-structure spectra}

The Balmer- $\alpha$ line of atomic hydrogen was optically thin in the plasma used in the present experiment. The magnitude of absorption was $\alpha_{0} l \simeq 1 \times 10^{-3}$. In spite of the weak absorption, we succeeded in measuring the Doppler-broadened absorption spectrum with the saturation dips by modulating the rf power and by using the lockin amplifier. The saturation spectra observed in the $\pi$ polarization configuration are shown in Fig. 4. Figure 4(a) was obtained at various distances from the electrode when the potential of the electrode was grounded. Figure 4(b) was obtained at a distance of $0.4 \mathrm{~mm}$ from the electrode surface when the voltage applied to the electrode was varied between 0 and $-120 \mathrm{~V}$ with respect to the ground potential. It is considered that spectrum (i) of Fig. 4(a), which was observed at a distance of $1.1 \mathrm{~mm}$ from the grounded electrode, corresponds to the field-free spectrum of the Balmer- $\alpha$ line of atomic hydrogen, since this measurement position was the outside of the sheath region. However, as reported in literature, there is a possibility that the spectrum (i) of Fig. 4(a) is affected slightly by the microfield (Holtsmark field) [21,22]. The labels shown in Fig. 4(a) correspond to the labels in the theoretical spectrum shown in Fig. 2. Peaks B, C, D, F, and G observed experimentally agreed well with the theoretical calculation. Peaks $\mathrm{A}$ and $\mathrm{E}$ were not detected because of the small amplitudes. Peaks with label X were not seen in the theoretical spectrum, but they are understood to be the cross over components which are unavoidable parasitic signals in saturation spectroscopy. The full widths at half maximum of the peaks were approximately $420 \mathrm{MHz}$. The theoretical value of $\Gamma_{\mathrm{s}}$ in Eq. (3) is determined by the natural broadening, the collisional broadening and the saturation broadening. The natural broadening width of the Balmer- $\alpha$ line is narrower than $110 \mathrm{MHz}$. The ion impact broadening width can be estimated to be approximately $50 \mathrm{MHz}$ in the plasma used in the experiment [33], and the broadening widths caused by collisions with electrons and neutral species are much narrower than the natural broadening. The additional broadening due to saturation, which is given by a factor of $\left(1+\sqrt{1+S_{0}}\right) / 2$, is also negligible because $S_{0} \simeq 0.1$ in the present experiment. Accordingly, the mechanism for the additional broadening that results in the experimental line width of $\Gamma_{\mathrm{s}}=420 \mathrm{MHz}$ has not been understood yet.

\subsection{Comparison between experimental and theoretical Stark spectra}

Firstly, we compare the experimental spectra shown in Fig. 4(a) with the theoretical Stark spectra. The frequencies of the $2 \mathrm{p}^{2} \mathrm{P}_{3 / 2}^{\mathrm{o}}-3 \mathrm{~d}^{2} \mathrm{D}_{3 / 2}$ (peak B) and $2 \mathrm{p}^{2} \mathrm{P}_{3 / 2}^{\mathrm{o}}-3 \mathrm{~d}^{2} \mathrm{D}_{5 / 2}$ (peak C) transitions shifted toward the low- and high-frequency sides, respectively, when we moved the observation position toward the vicinity to the electrode surface, as shown in Fig. 4(a). This result agreed with the theoretical spectra shown in Fig. 2(a). In addition, the shift of the $2 \mathrm{~s}^{2} \mathrm{~S}_{1 / 2}-3 \mathrm{p}^{2} \mathrm{P}_{1 / 2}^{\mathrm{o}}$ transition (peak $\mathrm{D}$ ) toward the low-frequency side was also observed at positions between 1.1 and $0.4 \mathrm{~mm}$ from the electrode. However, 


\section{Stark spectroscopy at Balmer- $\alpha$ line of atomic hydrogen for measuring sheath electric field in a hydrogen $p$}

peak D with Stark splitting was not detected experimentally at closer distances from the electrode because of the small amplitude. The shift of the $2 \mathrm{p}^{2} \mathrm{P}_{1 / 2}^{\mathrm{o}}-3 \mathrm{~d}^{2} \mathrm{D}_{3 / 2}$ (peak $\mathrm{G}$ ) transition toward the high frequency side, which is shown in the theoretical spectrum, was not observed clearly in the experimental spectrum shown in Fig. 4(a), which may be partly due to the broadening of the experimental spectrum. The broadening of peak $\mathrm{G}$ in the experimental spectrum may be due to the occurrence of multiple cross over components among the peaks originated from the Stark splitting.

Secondly, the experimental spectra shown in Fig. 4(b) is compared with the theoretical spectra at electric fields higher than $200 \mathrm{~V} / \mathrm{cm}$. The theoretical spectra shown in Fig. 2(a) clearly shows the splittings and shifts of peaks B and C, whereas in the experimental spectra, we observed a smaller number of peaks with significant broadenings. The mechanism of the broadening is the occurrence of many cross over components among the peaks originated from the Stark splitting. As a result, it was difficult to find clear peaks between 5 and $20 \mathrm{GHz}$ in the experimental spectra. On the other hand, we observed the splitting of peak $\mathrm{B}$ into two peaks $\left(\mathrm{B}_{1}\right.$ and $\left.\mathrm{B}_{2}\right)$ as indicated in Fig. 4(b). The peaks corresponding to the two peaks are indicated in the theoretical spectra shown in Fig. 2(a) using the same labels. The peaks $\mathrm{B}_{1}$ and $\mathrm{B}_{2}$ shifted toward the low-frequency side with the increase in the voltage applied to the electrode. In addition, the distance between peaks $\mathrm{B}_{1}$ and $\mathrm{B}_{2}$ increased with the applied voltage. These results agreed with the theoretical result.

The experimental Stark spectra observed in the $\sigma$ polarization configuration is shown in Fig. 5, which was observed at various distances from the grounded electrode. The broadening of peak $\mathrm{C}$ was observed at a close distance to the electrode surface, which is also seen in the theoretical spectra shown in Fig. 2(b). The variation of the spectrum around peaks $\mathrm{F}$ and $\mathrm{G}$ was also similar to the result of theoretical calculation. However, the splitting and the shift of peak D was not observed experimentally because of the small amplitude, which was a problematic result when we try to evaluate the electric field from the Stark spectra in the $\sigma$ polarization configuration.

\subsection{Measures to evaluate electric field}

The goal of Stark spectroscopy is to evaluate the electric field strength by the comparison between the experimental and theoretical spectra. For this purpose, it is necessary to pick up measures, which are useful to evaluate the electric field, from the Stark spectra. As can be understood from the comparison between Figs. 2(b) and 5, it was difficult to find a useful measure in the Stark spectra observed in the $\sigma$ polarization configuration. A measure we employed was the frequency of the $2 \mathrm{p}^{2} \mathrm{P}_{3 / 2}^{\mathrm{o}}-3 \mathrm{~d}^{2} \mathrm{D}_{3 / 2}$ (peak B) transition in the spectra observed in the $\pi$ polarization configuration. A reason why we chose this peak as the measure was that the shift of peak B was sensitive to the electric field. In addition, as shown in Fig. 4(b), the width of peak $\mathrm{B}_{2}$ was narrowest in the peaks observed in the high electric field. Figure 6 (a) shows the relationship between the position of peak $\mathrm{B}\left(\mathrm{B}_{2}\right)$ and the electric field strength, which was obtained by analyzing 
the theoretical Stark spectra. The position of peak B $\left(\mathrm{B}_{2}\right)$ in the experimental spectra is plotted in Fig. 6(b) as a function of the voltage applied to the electrode. The shift of peak B toward the low-frequency side indicates the increase in the electric field, and we can evaluate the electric field strength by comparing Fig. 6(b) with Fig. 6(a).

A problem of this measure was the ambiguity in the absolute frequency shift of peak B. This is because we used a Fabry-Pérot spectrum analyzer to measure the peak frequencies in the spectrum. The Fabry-Pérot spectrum analyzer does not give us the absolute frequency. The spacings among the peaks in the signal from the spectrum analyzer was $1 \mathrm{GHz}$, but the baseline of the frequency measurement shifted with time in the long experiment due to the change in the temperature. Therefore, the accurate determination of a small frequency shift of peak B was difficult in a low electric field. In this case, we employed the distance between peaks $\mathrm{B}$ and $\mathrm{C}$ as another measure to evaluate the weak electric field. The distance between peaks B and C was able to be determined accurately by comparing it with the spacing among the peaks in the signal from the spectrum analyzer. Figure 7(a) shows the distance between peaks B and C in the theoretical Stark spectra as a function of the electric field strength. The distance between peaks $\mathrm{B}$ and $\mathrm{C}$ in the experimental spectra is shown in Fig. 7(b) as a function of the distance from the grounded electrode. We can evaluate the electric field strength by comparing Fig. 7(b) with Fig. 7(a). The meaning of the horizontal error bars in Fig. 7(b) will be described in a later subsection.

\subsection{Evaluation of electric field}

The electric field strength is shown in Fig. 8(a) as a function of the distance from the grounded electrode. This was obtained by the comparison between Figs. 7(a) and 7(b). The distance between peaks B and $\mathrm{C}$ was used as the measure to evaluate the electric field strength. On the other hand, Fig. 8(b) shows the relationship between the electric field strength at a distance of $0.4 \mathrm{~mm}$ from the electrode and the voltage applied to the electrode. This was obtained by the comparison between Figs. 6(a) and 6(b).

\subsection{Spatial resolution}

The spatial resolution is an important performance when we apply the developed method to the measurement of the spatial distribution of the electric field in the sheath region. The ambiguity in the measurement position is caused by the finite diameters of the pump and probe beams. In the following evaluation of the spatial resolution, it is assumed that the pump and probe beams have the same profile and are overlapped perfectly. This condition is almost realized in the experiment. We suppose that the resonance frequency of the transition line has a variation within the size of the laser beam, since the electric field has a gradient along the distance $\mathrm{z}$ from the electrode surface. Under the assumption that the pump and probe beams have Gaussian profiles with a diameter 
(FWHM) $w_{0}$, the signal intensity of the saturation spectrum becomes

$$
\begin{aligned}
\Delta I_{\mathrm{t}}(\nu, z) & =\Delta I_{0} \frac{\left(\gamma_{\mathrm{s}} / 2\right)^{2}}{\left(\nu-\nu_{0}(z)\right)^{2}+\left(\Gamma_{\mathrm{s}} / 2\right)^{2}} \int_{\infty}^{\infty} \exp \left(-8(\ln 2) \frac{z^{2}+x^{2}}{w_{0}^{2}}\right) \mathrm{d} x \\
& =\frac{\Delta I_{0} w_{0}}{2} \sqrt{\frac{\pi}{2(\ln 2)}} \frac{\left(\gamma_{\mathrm{s}} / 2\right)^{2}}{\left(\nu-\nu_{0}(z)\right)^{2}+\left(\Gamma_{\mathrm{s}} / 2\right)^{2}} \exp \left(-8(\ln 2) \frac{z^{2}}{w_{0}^{2}}\right)
\end{aligned}
$$

where $\Delta I_{0}$ is the proportional constant which gives the amplitude of the peak, since $\Delta I_{\mathrm{t}}(\nu) \propto I_{\mathrm{i}} S_{0} \propto I_{\mathrm{i}} I_{\mathrm{p}}$ and $\alpha_{0}(\nu)$ is almost constant within the linewidth $\Gamma_{\mathrm{s}}$. In Eq. (4), $\mathrm{x}$ represents the distance from the center on the cross section of the laser beams in the parallel direction to the target surface. For the sake of simplicity, we analyze the case where $\nu_{0}(z)$ has a linear variation with $\mathrm{z}$ such that

$$
\nu_{0}(z)=\nu_{0}(0)+\zeta z
$$

where $\zeta$ represents the slope of the resonance frequency. We analyzed the profile of $\Delta I_{\mathrm{t}}\left(\nu_{0}(0), z\right)$ by substituting the experimental parameters of $w_{0}=0.3 \mathrm{~mm}, \Gamma_{\mathrm{s}}=420$ $\mathrm{MHz}$, and $\zeta=1.5 \mathrm{GHz} / \mathrm{mm} . \zeta=1.5 \mathrm{GHz} / \mathrm{mm}$ was observed in the sheath region at a distance of $z \leq 0.3 \mathrm{~mm}$ from the grounded electrode, as shown in Fig. 4(a). As a result, we found that the full width at half maximum of $\Delta I_{\mathrm{t}}\left(\nu_{0}(0), z\right)$ was $0.15 \mathrm{~mm}$. This value gives us the spatial resolution of the developed method, and is shown by the horizontal error bars in Figs. 7(b) and 8(a). At a longer distance $(z>0.3 \mathrm{~mm})$ from the electrode, the spatial resolution was evaluated to be $0.2 \mathrm{~mm}$ because of the smaller value of $\zeta$. It is noted that the spatial resolution is sensitive to $\zeta$. A better spatial resolution is obtained in the sheath with a steeper gradient of the electric field.

\subsection{Detection limit and ambiguity}

Although the saturation spectrum has a linewidth of $420 \mathrm{MHz}$, we can identify much smaller shifts of the peak positions of the transition lines. The minimum change in the distance between peaks B and C, which we could identify experimentally, was $10 \mathrm{MHz}$. According to Fig. 7(a), the change of $10 \mathrm{MHz}$ in the frequency distance between peaks $\mathrm{B}$ and $\mathrm{C}$ corresponds to the electric field of $10 \mathrm{~V} / \mathrm{cm}$. Therefore, the minimum electric field which can be detected by the developed method is evaluated to be $10 \mathrm{~V} / \mathrm{cm}$. This is a more sensitive detection limit than those of conventional laser optogalvano spectroscopy and laser-induced collisional fluorescence spectroscopy. Although laserinduced fluorescence-dip spectroscopy has a better detection limit, a sensitivity higher than $10 \mathrm{~V} / \mathrm{cm}$ is meaningless in practical plasma diagnostics, since it was observed that the static electric field was masked by microfields $[21,22]$. Therefore, the detection limit of $10 \mathrm{~V} / \mathrm{cm}$ is sufficient when we apply the developed method to the diagnostics of lowtemperature plasmas. The ambiguity in the measured electric field is also influenced by the ambiguity in the determination of the peak positions. When the electric field is deduced from the distance between peaks $\mathrm{B}$ and $\mathrm{C}$, the ambiguity in the frequency distance is $10 \mathrm{MHz}$, corresponding to the ambiguity of $10 \mathrm{~V} / \mathrm{cm}$ in the electric field. When the electric field is deduced from the position of peak $\mathrm{B}\left(\mathrm{B}_{2}\right)$, the ambiguity in 
the frequency determination is $40 \mathrm{MHz}$, corresponding to the ambiguity of $30 \mathrm{~V} / \mathrm{cm}$ in the electric field. The ambiguity of $10 \mathrm{~V} / \mathrm{cm}$ is indicated by the vertical error bars in Fig. 8(a). Since other ambiguities are smaller than the plot symbols, the vertical error bars are not indicated in Figs. 6(b), 7(b), and Fig. 8(b).

\subsection{Limitation in developed method}

A difficulty of the developed method is caused by the fact that the Balmer- $\alpha$ line of atomic hydrogen is optically thin in many low-temperature plasmas. In this work, we amplified the absorption spectrum by modulating the plasma production and by using a lock-in amplifier. However, the pulse modulation of the plasma production is not applicable in many experimental conditions. An alternative method is modulating the pump laser beam using an optical chopper or an electro-optic modulator, and operating the lock-in amplifier with the reference signal at the frequency of the optical modulation. We succeeded in measuring Stark spectra in a cw hydrogen plasma by the optical modulation of the pump beam. Similar spectra were obtained but the signal-to-noise ratio was slightly lower than that obtained by the modulation of the plasma production. Another possibility is to employ an absorption line from a metastable state of argon or helium. In this case, we can observe the saturation spectrum with a much higher sensitivity. However, the Stark effects of the low-energy states of argon and helium were much less sensitive to a weak electric field than the Balmer- $\alpha$ line of atomic hydrogen.

Another limitation of the developed method could be caused by the fact that the electric field is deduced from the spectrum observed in the $\pi$ polarizaion configuration. If the direction of the electric field is unknown, we observe the superposition of the Stalk spectra in the $\pi$ and $\sigma$ polarization configurations. In this case, we expect that the component of the $\sigma$ polarization configuration can be eliminated by rotating the polarization of the pump and probe beams using half-wavelength plates. We expect that the direction of the electric field is estimated by comparing the Stark spectra observed at various polarization directions of the laser beams.

\section{Conclusions}

In this paper, we have described a method for measuring the sheath electric field in a low-temperature hydrogen plasma. The method is based on the measurement of the Stark spectrum of the Balmer- $\alpha$ line of atomic hydrogen by saturation spectroscopy with a Doppler-free spectral resolution. The detection limit of $10 \mathrm{~V} / \mathrm{cm}$ has been realized, which is a sufficient sensitivity for investigating the structures of the sheath electric fields in low-temperature plasmas. Shifting the approach from a Rydberg state to a low-energy state enables us to use an economical single-mode diode laser system for the electric field measurement. The less sensitive Stark effect of the low-energy state is compensated by the ultrafine resolution of saturation spectroscopy. In addition, since the diode laser system is easy to use, the developed system is useful in various experiments which need 
Stark spectroscopy at Balmer- $\alpha$ line of atomic hydrogen for measuring sheath electric field in a hydrogen $p$

the measurements of electric fields in plasmas.

\section{Acknowledgments}

This work is performed with the support and under the auspices of the NIFS Collaboration Research Program (NIFS15KLER042).

\section{References}

[1] Hershkowitz N 2005 Phys. Plasmas 12055502

[2] Okuno Y and Fujita H 1991 J. Appl. Phys. 70642

[3] Schwager L A, Hsu W L and Tung D M 1993 Phys. Fluids B 5621

[4] Hershkowitz N 1994 IEEE Trans. Plasma Sci. 2211

[5] Oksuz L and Hershkowitz N 2002 Phys. Rev. Lett 89145001

[6] Oksuz L, Khedr M A and Hershkowitz N 2001 Phys. Plasmas 81729

[7] Severn G D, Wang X, Ko E and Hershkowitz N 2003 Phys. Rev. Lett 90145001

[8] Lee D, Hershkowitz N and Severn G D 2007 Appl. Phys. Lett 91041505

[9] Hebner G A and Paterson A M 2010 Plasma Sources Sci. Technol. 19015020

[10] Doughty D K and Lawler J E 1984 Appl. Phys. Lett. 45611

[11] Ganguly B N, Shoemaker J R, Preppernau B L and Garsccadden A 1987 J. Appl. Phys. 612778

[12] Preppernau B L and Ganguly B N 1993 Rev. Sci. Instrum. 641414

[13] Gavrilenko V P, Kim H J, Ikutake T, Kim J B, Choi T W, Bowden M D and Muraoka K 2000 Phys. Rev. E 627201

[14] Greenberg K E and Hebner G A 1993 Appl. Phys. Lett. 633282

[15] Booth J P, Fadlallah M, Derouard J and Sadeghi N 1994 Appl. Phys. Lett 65819

[16] Bowden M D, Choi Y W, Muraoka K and Maeda M 1995 Appl. Phys. Lett. 661059

[17] Choi Y W, Bowden M D and Muraoka K 1996 Appl. Phys. Lett. 691361

[18] Kim J B, Kawamura K, Choi Y W, Bowden M D, Muraoka K and Helbig V 1998 IEEE Trans. Plasma Sci. 261556

[19] Czarnetzki U, Luggenhölscher D and Döbele H F 1998 Phys. Rev. Lett. 814592

[20] Kampschulte T, Schulze J, Luggenhl̈scher D, Bowden M D, and Czarnetzki U 2007 New J. Phys. 918

[21] Takizawa K, Sasaki K and Kono A 2004 Appl. Phys. Lett. 84185

[22] Takizawa K, Kono A, and Sasaki K 2007 Appl. Phys. Lett. 90011503

[23] Takizawa K and Sasaki K 2007 Jpn. J. Appl. Phys. 466822

[24] Barnat E V and Hebner G A 2004 Appl. Phys. Lett. 853393

[25] Barnat E V and Hebner G A 2004 J. Appl. Phys. 964762

[26] Wagenaars E, Kroesen G M W and Bowden M D 2006 Phys. Rev. A 74033409

[27] Booth J P, Derouard J, Fadlallah M, Cabaret L and Pinard J 1996 Opt. Comm. 132363

[28] Adamov M G, Steiger A, Grëzmacher K and Seidel J 2007 Phys. Rev. A 75013409

[29] Asakawa R, Goto M, Sadeghi N and Sasaki K 2012 J. Instrum. 7 C01018

[30] Nishiyama S, Katayama K, Nakano H, Goto M, and Sasaki K 2017 Appl. Phys. Express 10036101

[31] Demtröder W 1998 Laser Spectroscopy 2nd ed. (Springer, Berlin)

[32] Fujimoto T and Iwamae A (Editors) 2008 Plasma Polarization Spectroscopy (Springer, Berlin)

[33] Fujimoto T 2004 Plasma Spectroscopy (Oxford University Press, New York) 
Stark spectroscopy at Balmer- $\alpha$ line of atomic hydrogen for measuring sheath electric field in a hydrogen $p$

\section{Figure captions}

Fig.1 Energy level diagram of atomic hydrogen relevant to the Balmer- $\alpha$ line. Labels A-G correspond to those indicated in Figs. 2, 4, and 5.

Fig.2 Theoretical Stark spectra of the Balmer- $\alpha$ line of atomic hydrogen at various electric fields. (a) The polarizations of the pump and probe laser beams are parallel to the electric field ( $\pi$ polarization configuration). (b) The polarizations of the pump and probe laser beams are perpendicular to the electric field ( $\sigma$ polarization configuration).

Fig.3 Schematic of experimental apparatus.

Fig.4 Experimental Stark spectra observed in the $\pi$ polarization configuration. (a) was observed at various distances from the surface of the grounded electrode. (b) was observed at a distance of $0.4 \mathrm{~mm}$ from the surface of the electrode when it was connected to the dc power supply at various voltages.

Fig.5 Experimental Stark spectra observed in the $\sigma$ polarization configuration at various distances from the surface of the grounded electrode.

Fig.6 Frequency of the $2 \mathrm{p}^{2} \mathrm{P}_{3 / 2}^{\mathrm{o}}-3 \mathrm{~d}^{2} \mathrm{D}_{3 / 2}$ transition (peak $\mathrm{B}$ (or $\mathrm{B}_{2}$ )) in the $\pi$ polarization configuration. (a) is the theoretical result as a function of the electric field. (b) is the experimental result observed at a distance of $0.4 \mathrm{~mm}$ from the electrode as a function of the applied voltage.

Fig. 7 Frequency distance between the $2 \mathrm{p}^{2} \mathrm{P}_{3 / 2}^{\mathrm{o}}-3 \mathrm{~d}^{2} \mathrm{D}_{3 / 2}$ (peak B) and $2 \mathrm{p}^{2} \mathrm{P}_{3 / 2}^{\mathrm{o}}-3 \mathrm{~d}^{2} \mathrm{D}_{5 / 2}$ (peak C) transitions in the $\pi$ polarization configuration. (a) is the theoretical result as a function of the electric field. (b) is the experimental result as a function of the distance from the surface of the grounded electrode.

Fig.8 Electric field strength evaluated by the developed method. (a) shows the electric field as a function of the distance from the surface of the grounded electrode. (b) shows the electric field at a distance of $0.4 \mathrm{~mm}$ from the electrode as a function of the applied voltage. 
Stark spectroscopy at Balmer- $\alpha$ line of atomic hydrogen for measuring sheath electric field in a hydrogen $p$

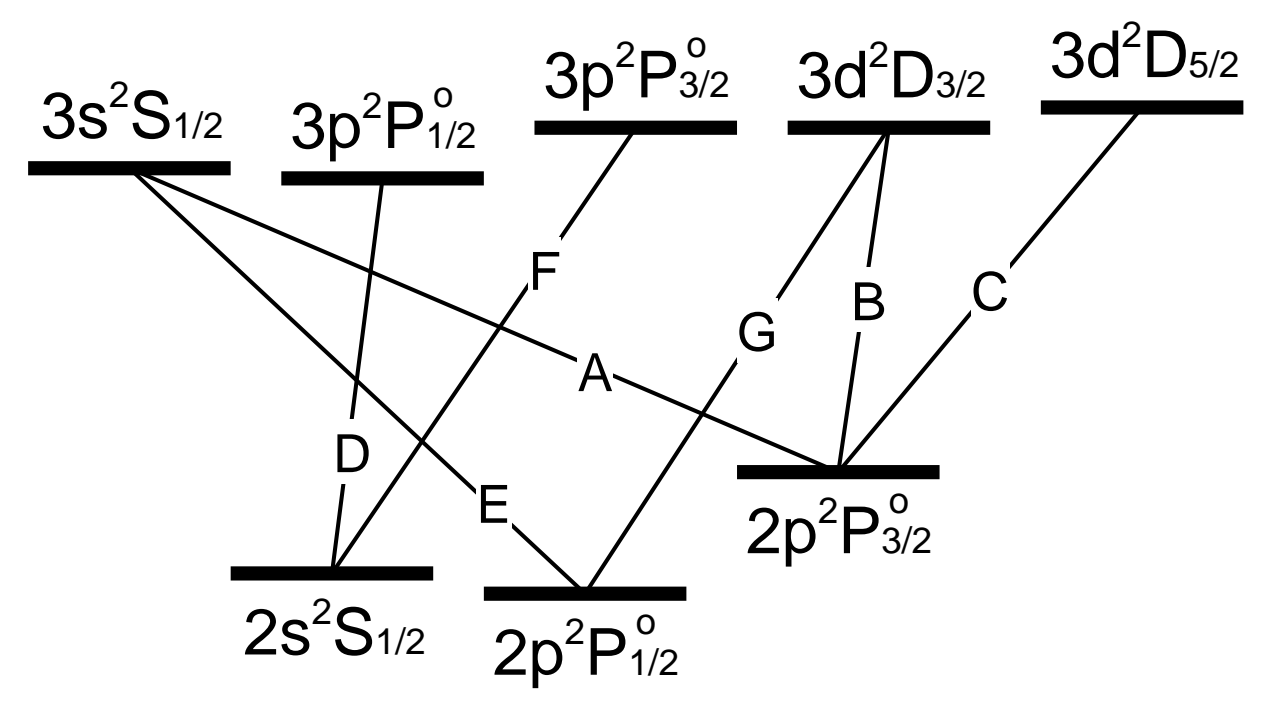

Figure 1. Energy level diagram of atomic hydrogen relevant to the Balmer- $\alpha$ line. Labels A-G correspond to those indicated in Figs. 2, 4, and 5. 

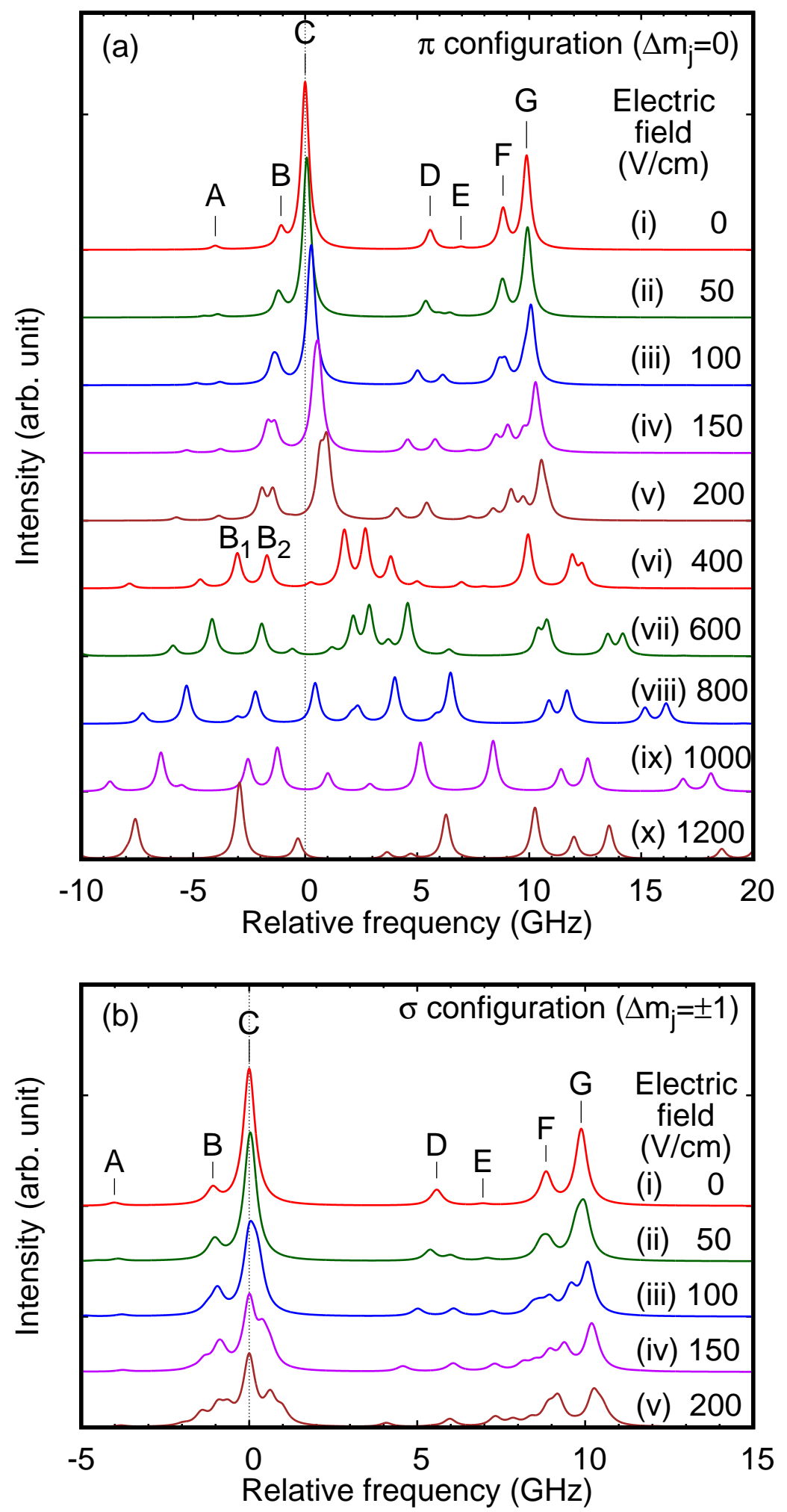

Figure 2. Theoretical Stark spectra of the Balmer- $\alpha$ line of atomic hydrogen at various electric fields. (a) The polarizations of the pump and probe laser beams are parallel to the electric field ( $\pi$ polarization configuration). (b) The polarizations of the pump and probe laser beams are perpendicular to the electric field ( $\sigma$ polarization configuration). 


\section{Stark spectroscopy at Balmer- $\alpha$ line of atomic hydrogen for measuring sheath electric field in a hydrogen $p$}

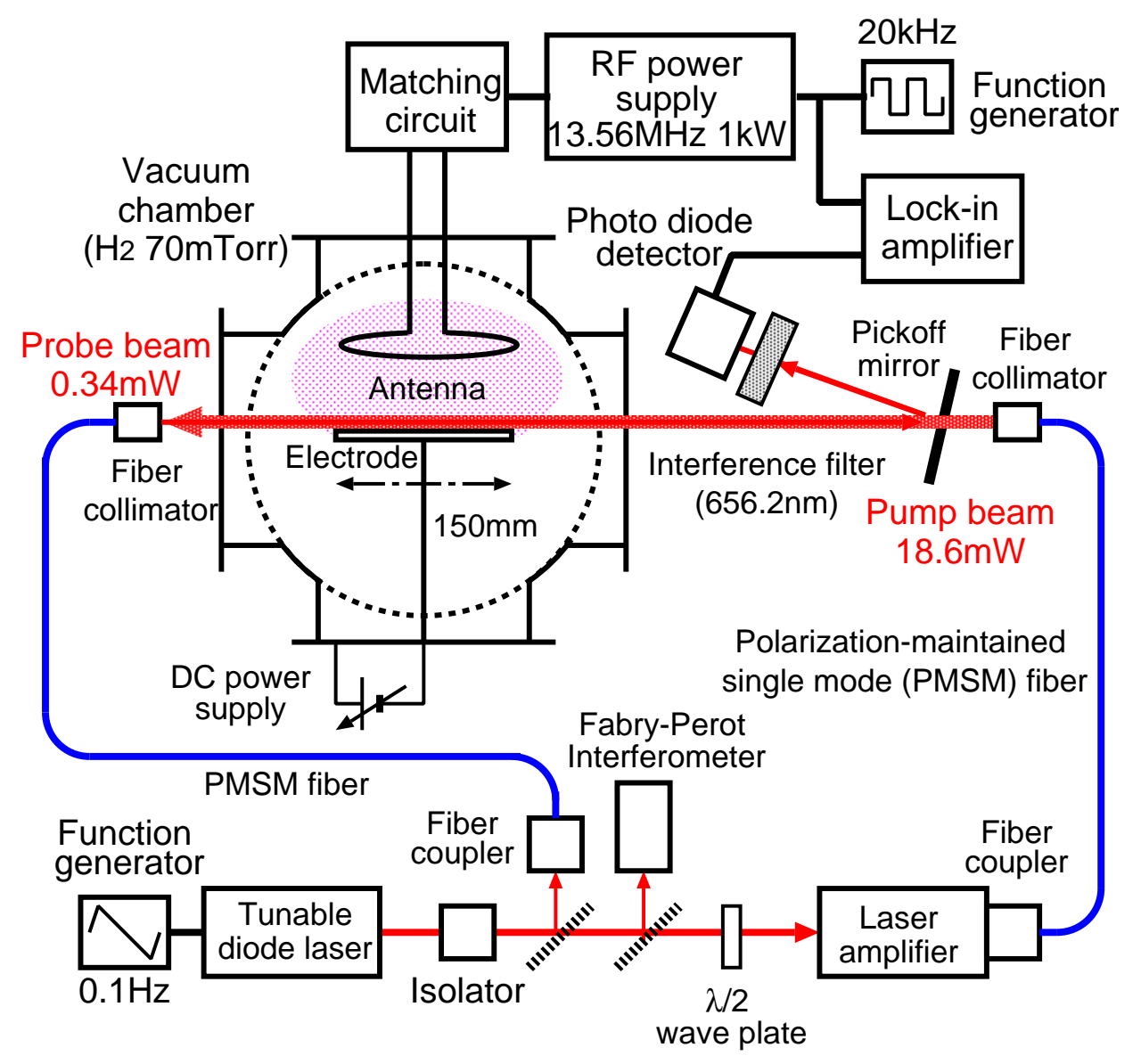

Figure 3. Schematic of experimental apparatus. 

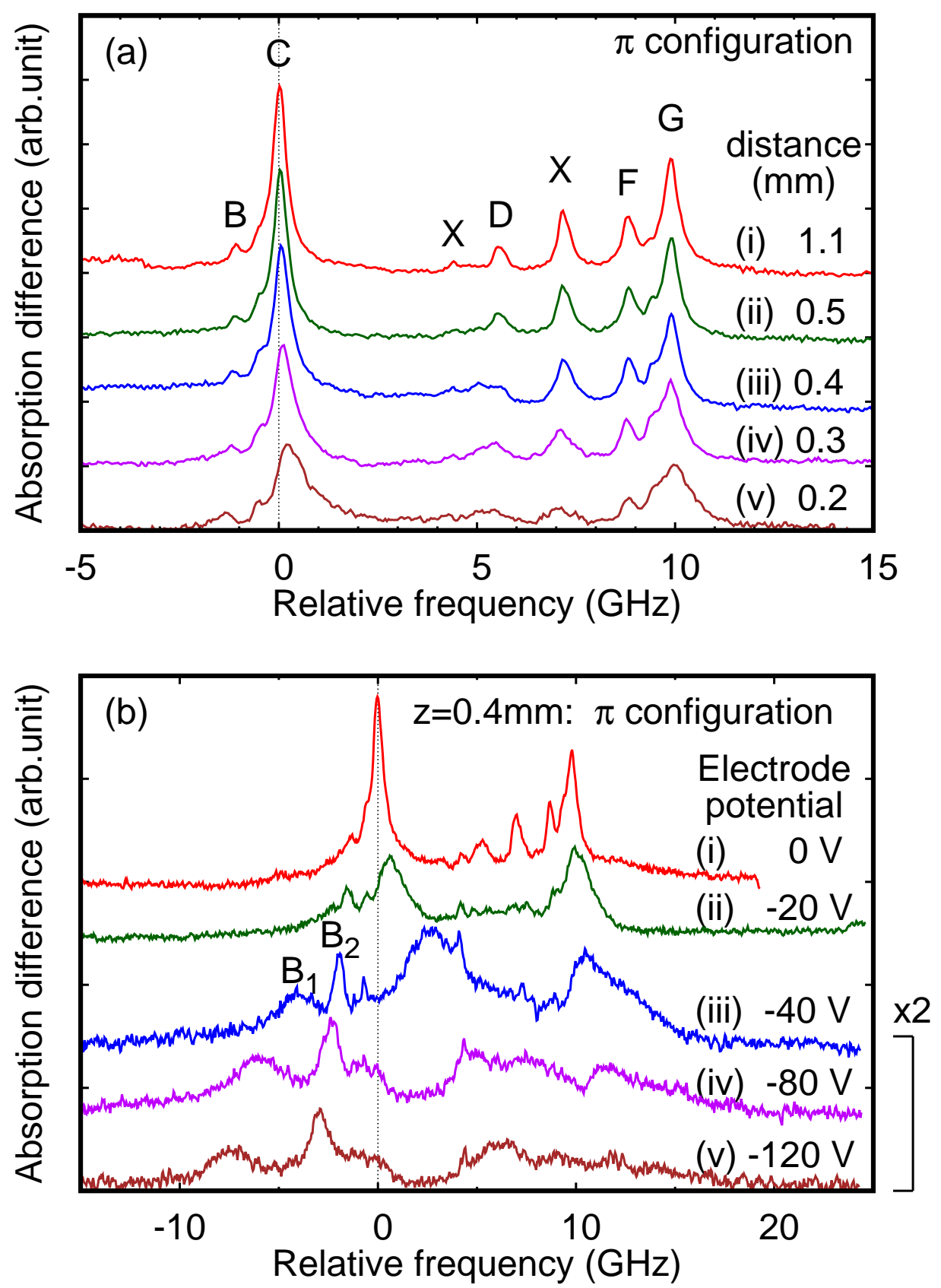

Figure 4. Experimental Stark spectra observed in the $\pi$ polarization configuration. (a) was observed at various distances from the surface of the grounded electrode. (b) was observed at a distance of $0.4 \mathrm{~mm}$ from the surface of the electrode when it was connected to the dc power supply at various voltages. 
Stark spectroscopy at Balmer- $\alpha$ line of atomic hydrogen for measuring sheath electric field in a hydrogen $p$

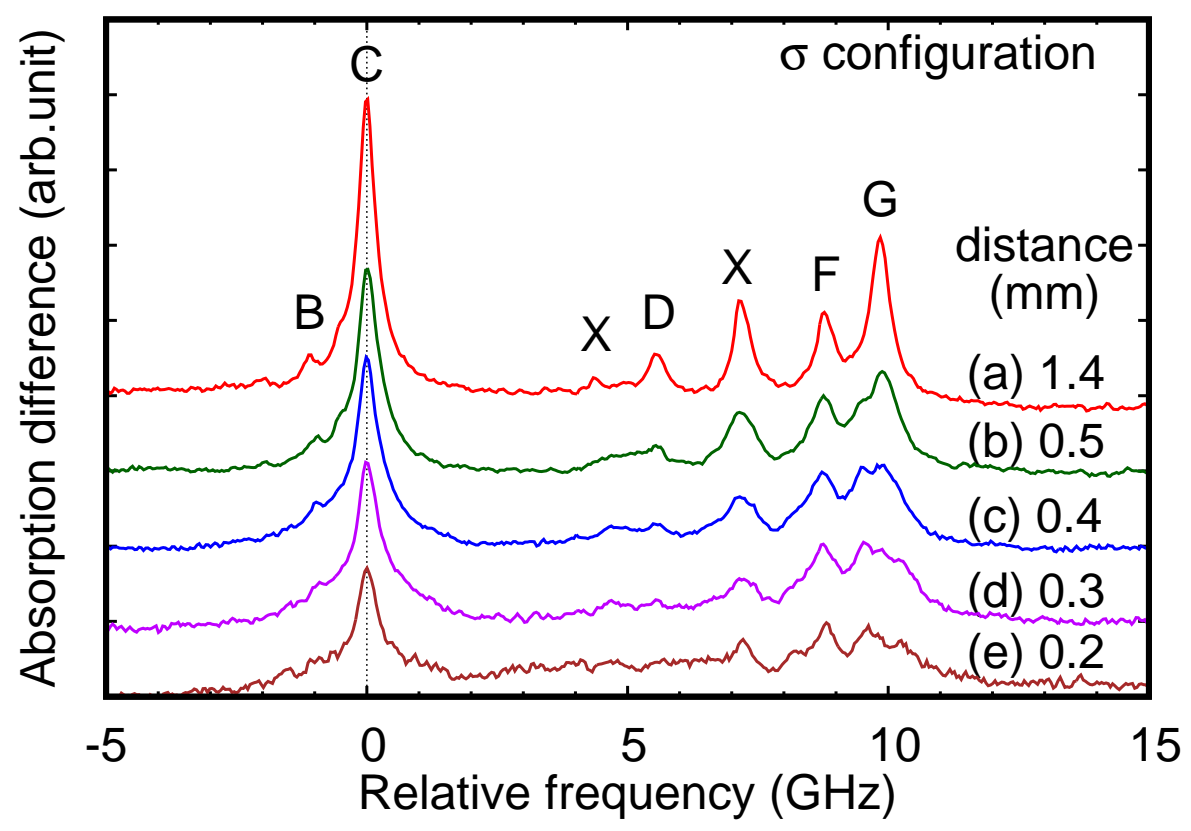

Figure 5. Experimental Stark spectra observed in the $\sigma$ polarization configuration at various distances from the surface of the grounded electrode. 

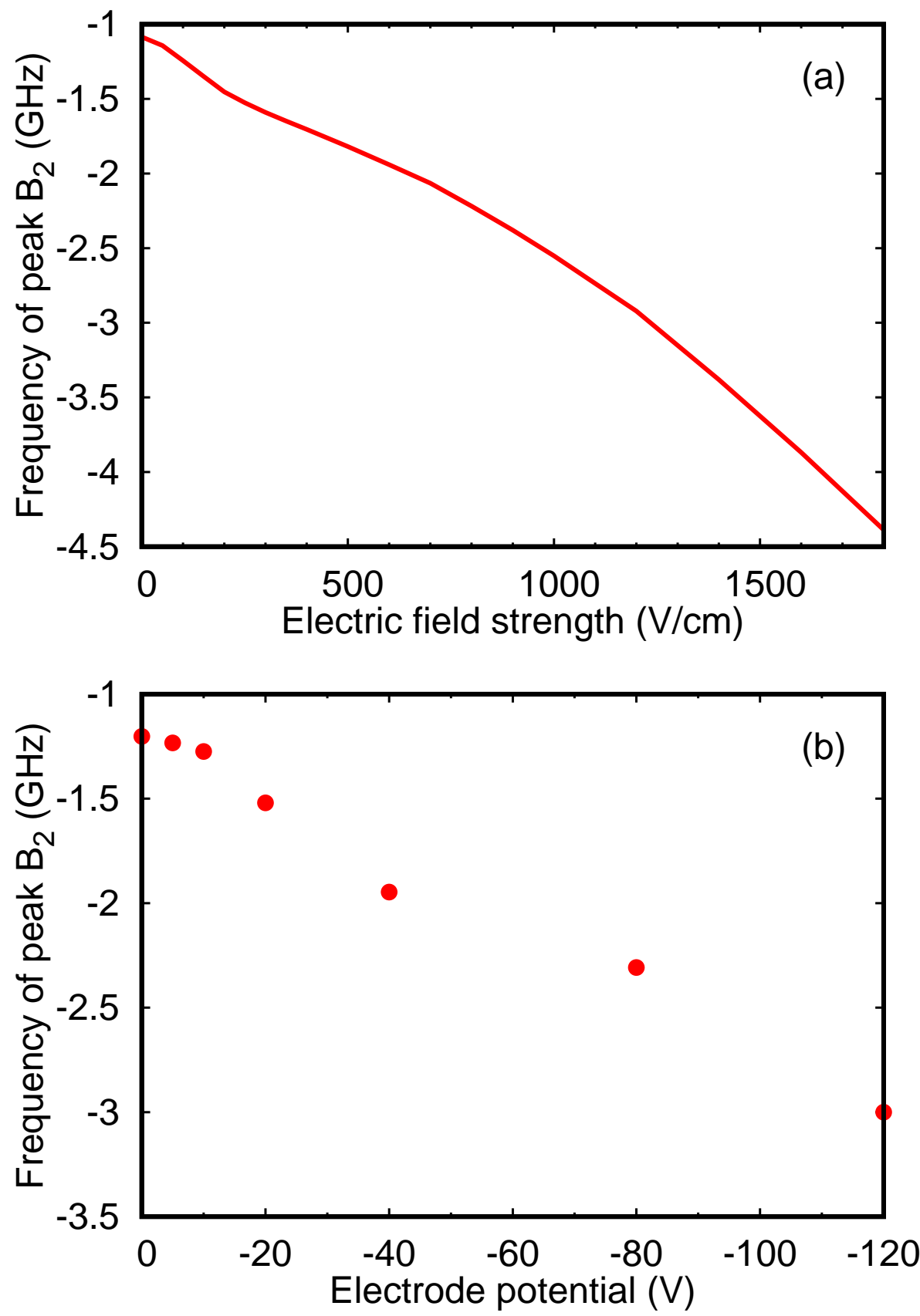

Figure 6. Frequency of the $2 \mathrm{p}^{2} \mathrm{P}_{3 / 2}^{\mathrm{o}}-3 \mathrm{~d}^{2} \mathrm{D}_{3 / 2}$ transition (peak $\mathrm{B}\left(\right.$ or $\mathrm{B}_{2}$ )) in the $\pi$ polarization configuration. (a) is the theoretical result as a function of the electric field. (b) is the experimental result observed at a distance of $0.4 \mathrm{~mm}$ from the electrode as a function of the applied voltage. 


\section{Stark spectroscopy at Balmer- $\alpha$ line of atomic hydrogen for measuring sheath electric field in a hydrogen $p$}
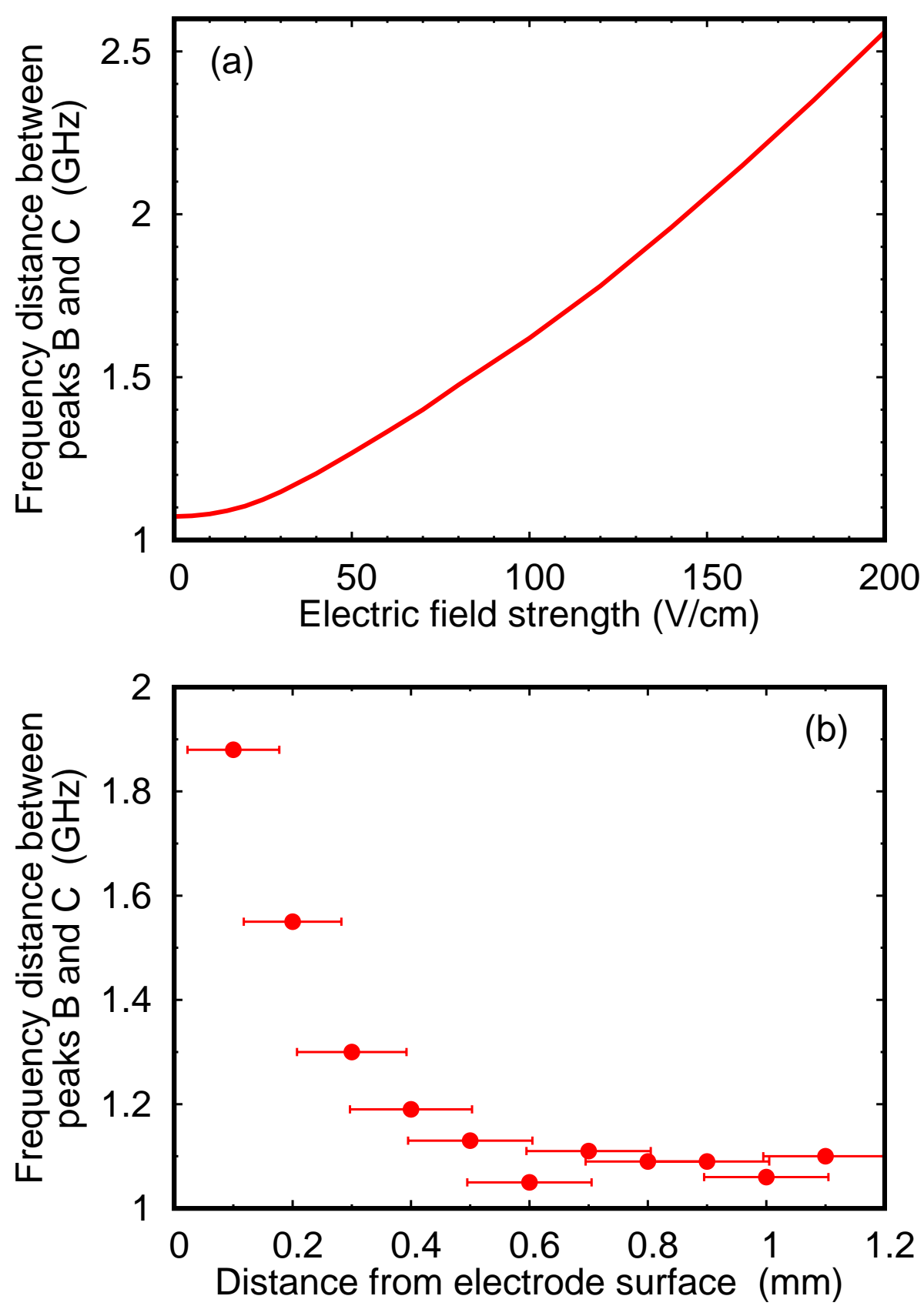

Figure 7. Frequency distance between the $2 \mathrm{p}^{2} \mathrm{P}_{3 / 2}^{\mathrm{o}}-3 \mathrm{~d}^{2} \mathrm{D}_{3 / 2}$ (peak B) and $2 \mathrm{p}^{2} \mathrm{P}_{3 / 2}^{\mathrm{o}}-3 \mathrm{~d}^{2} \mathrm{D}_{5 / 2}$ (peak C) transitions in the $\pi$ polarization configuration. (a) is the theoretical result as a function of the electric field. (b) is the experimental result as a function of the distance from the surface of the grounded electrode. 

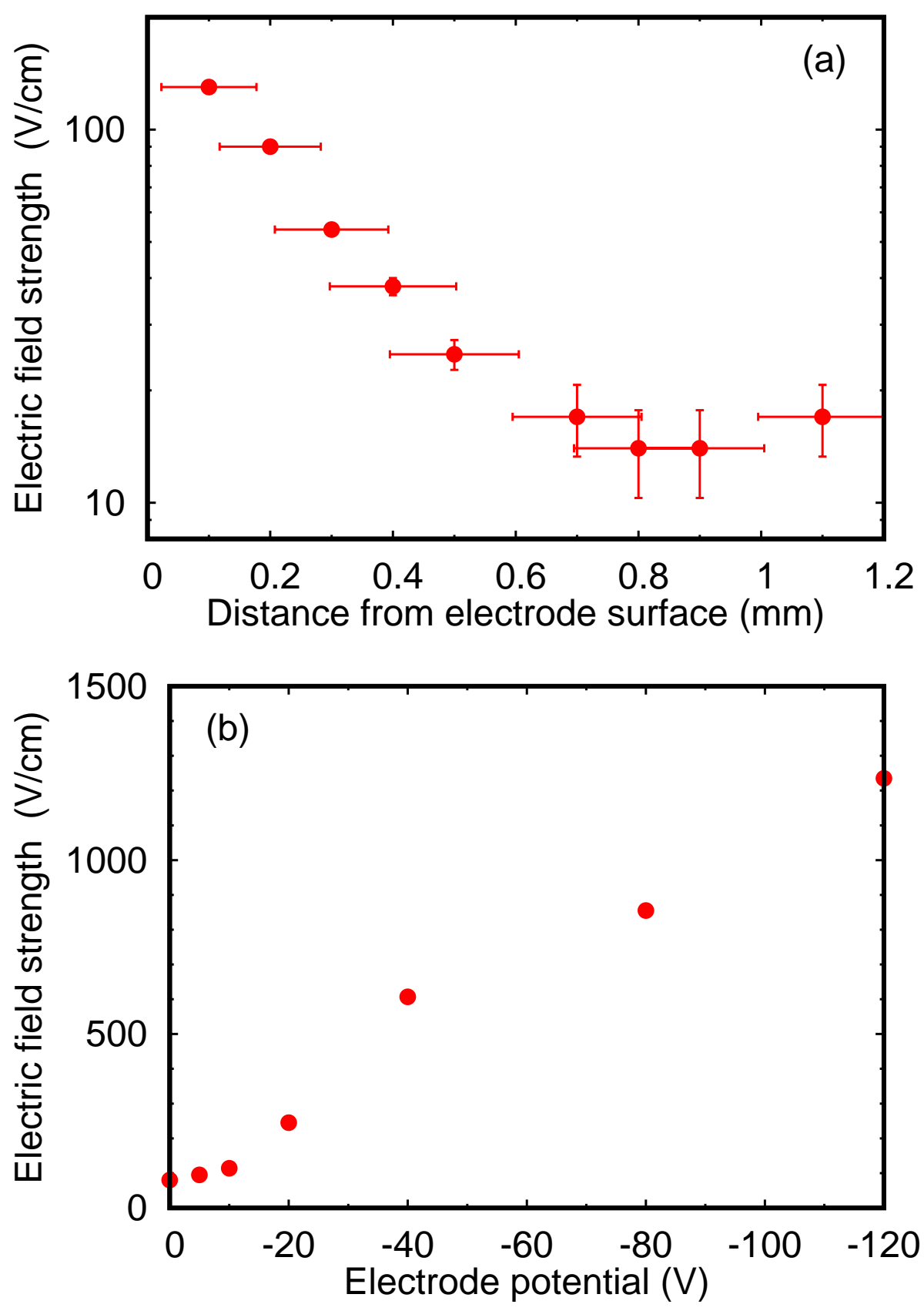

Figure 8. Electric field strength evaluated by the developed method. (a) shows the electric field as a function of the distance from the surface of the grounded electrode. (b) shows the electric field at a distance of $0.4 \mathrm{~mm}$ from the electrode as a function of the applied voltage. 